\title{
Elastic shear buckling capacity of the longitudinally stiffened flat panels
}

\author{
S. Kitarovic, J. Andric \& K. Piric \\ Faculty of Mechanical Engineering and Naval Architecture, University of Zagreb, Zagreb, Croatia
}

\begin{abstract}
Various feasible approaches to the increase of the flat plate elastic shear buckling capacity are investigated. Based on derived theoretical envelopes of the considered approaches, stiffening parallel to the longer plate edges is identified as the most effective approach to the considered problem. An accompanying analytical formulation is approximated in a form convenient for utilization in structural analysis and design of the plated structures. All derived conclusions and proposed formulations are based on results of the numerous rationally designed numerical simulations utilizing the finite element method.
\end{abstract}

\section{INTRODUCTION}

\subsection{General remarks}

During the ship exploitation, an imposed vertical shear loading is predominantly resisted by the vertically oriented parts of the hull girder structure (e.g. sides and longitudinal bulkheads). Pronounced shear loading of the significant intensity can induce occurrence of the shear buckling and eventual shear collapse of the structural elements loaded in shear when acting shear load surpasses their ultimate shear load capacity. Possibility of incidence for such a hazardous event becomes even more significant if relevant structural elements have been damaged previously (e.g. by collision) and consequently suffered a notable decrease in the load capacity with respect to the undamaged condition. In this context, consideration of the shear load capacity of the hull girder structural elements can represent a relevant aspect and an important structural adequacy criterion in analysis and design of the ship structures.

Occurrence of the shear buckling does not represent the ultimate limit state of the plating loaded in edge shear. Its ultimate limit state is eventually reached by a total depletion of the complete load capacity margin of the diagonal tension fields, see Bleich \& Ramsey (1952), i.e. by the complete plastification (yielding) of the plate material loaded in tension. Unfortunately, exact assessment of the (ultimate) shear load capacity of the hull girder plating necessitates detailed description of the still unresolved and very complex interaction of all relevant parameters of influence. This disables complete and accurate theoretical description of the shear collapse phenomena. Consequently, virtually all of the existing ultimate shear strength formulations are of the semi-empirical nature and are predominantly formulated by means of the regression analyses based on various results obtained by experimental investigations and/or numerical simulations. Concise description of the various existing formulations of the plate ultimate shear load capacity can be found in Zhang et al. (2008).

Since the most of the contemporary ultimate shear load capacity formulations are based on the correction of the calculated elastic shear buckling critical stress, within the framework of this paper various possible approaches to the elastic shear buckling inhibition are discussed. The most obvious one among them is to maximize the rotational restraint along the plate edges, resulting ultimately with the clamped edge restraints. However, the actual edge restraint imposed at the plate edge joints varies between the two extreme cases (simply supported and clamped). Only the worst (simply supported) case, which provides the highest margin for the increase of the plate elastic shear load capacity, is considered further in this paper.

\subsection{Elastic shear buckling of the simply supported flat plates}

An isotropic, flat plate of length $L$, breadth $B$ and thickness $t$, loaded along the all four boundaries (edges) by pure and uniformly distributed in-plane edge shear stress is considered (see Fig. 1).

If the geometrical and material characteristics of the considered plate are such that elastic shear buckling occurs at the critical intensity of the imposed edge shear loading, the corresponding deflected state of the unstable equilibrium can be described by the St. Venants equation, see Timoshenko \& Gere (1985), (given for the pure edge shear case), which is valid if the lateral displacements are small with respect to $t$ : 


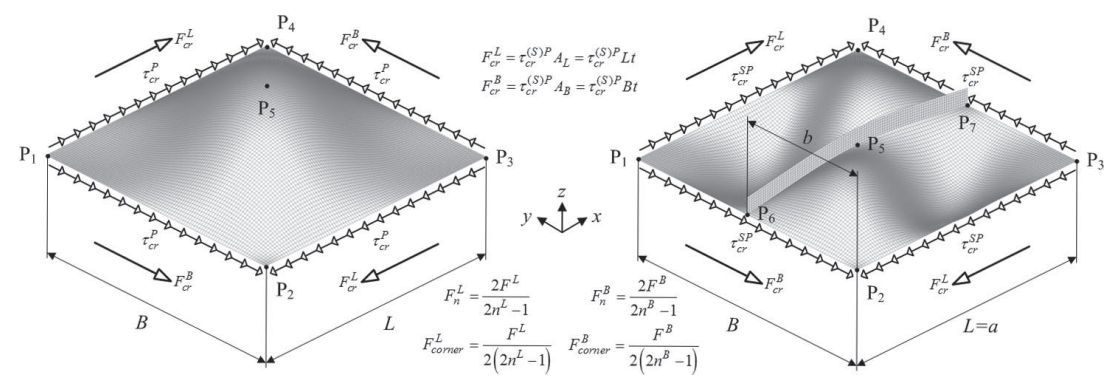

Figure 1. Elastic shear buckling of the simply supported (un)stiffened plate.

$$
\left(\frac{\partial^{4} w}{\partial x^{4}}+2 \frac{\partial^{4} w}{\partial x^{2} \partial y^{2}}+\frac{\partial^{4} w}{\partial y^{4}}\right)=-\frac{2 \tau_{c r}^{P} t}{D} \frac{\partial^{2} w}{\partial x \partial y}
$$

where $\tau_{c r}{ }^{P}$ represents the plate elastic shear buckling stress, $D$ denotes the plate flexural rigidity, while $w$ denotes the out-of-plane or lateral (in direction of the $z$-axis) displacement. Although the exact solution of (1) is not known, an approximate solution can be derived using the stationary potential energy principle and a Ritz method (lateral displacements expressed in terms of the appropriate coordinate functions which satisfy considered boundary conditions):

$$
\tau_{c r}^{P}=k^{P} \frac{\pi^{2} D}{B^{2} t}=k^{P} \frac{\pi^{2} E}{12\left(1-v^{2}\right)}\left(\frac{t}{B}\right)^{2}
$$

$E$ and $v$ denote Young's modulus of elasticity and Poisson's ratio, respectively, while $k^{P}$ denotes the non-dimensional coefficient dependant only on $L / B$ :

$$
k^{P}=\left\{\begin{array}{lll}
5.34+4\left(\frac{L}{B}\right)^{-2} & \text { for } & \frac{L}{B} \geq 1 \\
5.34\left(\frac{L}{B}\right)^{-2}+4 & \text { for } & \frac{L}{B}<1
\end{array}\right.
$$

Although formulation of $k^{P}$ given by (3) is widely accepted in the contemporary structural analysis and design practice, it actually represents the parabolic approximation of the more accurate values of $k^{P}$ calculated previously by various authors, see Bleich \& Ramsey (1952).

\subsection{Plate shear buckling capacity magnification}

It should be noted that for a given plate material, $\tau_{c r}{ }^{P}$ depends solely on the plate geometrical characteristics $L / B$ and $t / B$, according to (2). This suggests that $\tau_{c r}{ }^{P}$ can be increased by two distinct approaches: by decrease in the $L / B$ value and/or by increase in the $t / B$ value. Decrease in the $L / B$ value can be accomplished by subdivision of the plate onto smaller and equal parts by addition of the equidistantly placed transverse (parallel with the $y$-axis) stiffeners, whereby $t / B$ ratio remains unchanged. On the other hand, increase in the $t / B$ value can be accomplished by the increase of the plate thickness and/or by subdivision of the plate onto smaller and equal parts by addition of the equidistantly placed longitudinal (parallel with $x$-axis) stiffeners. Yet, since the aspect ratio of the obtained plate subdivisions $a / b$ (where $a$ denotes length of the plating between stiffeners - parallel to the stiffener orientation, while $b$ denotes breadth of the plating between stiffeners perpendicular to the stiffener orientation, see Figure 1) is thereby increased, it seems, at the first glance, that the positive effect of this approach is somewhat attenuated. Since various possible approaches to the plate elastic shear buckling capacity magnification are identified, it would be useful to determine the most effective one among them, i.e. to identify the most gainful approach which would enable maximum increase in critical shear stress.

In this respect, it is important to emphasize that the most gainful effects of the plate stiffening can be exploited only if the critical value of the acting shear load induces imminent occurrence of the local shear buckling (shear buckling of the plating between stiffeners), i.e. that occurrence of the global shear buckling (shear buckling of the whole stiffened plate) is successfully disabled. In another words, this will be accomplished only if the plating between stiffeners can be considered as a set of an independent, simply supported plates loaded in edge shear. Ability to comply with this requirement depends on the flexural rigidity of stiffener(s) employed, which must be sufficient to enable successful resistance of the stiffener(s) to the predominant vertical bending load imposed by the buckling half waves during their spread along the stiffened plate. 
Based on this paradigm, originally introduced by Timoshenko, see Bleich \& Ramsey (1952), Figure 2 illustrates the obtained maximum relative increases in the critical shear stress attainable by the longitudinal and/or transverse (equidistant) stiffening of the plate for various $L / B$ ratios and number of added stiffeners $N_{S}$, where $\tau_{c r}{ }^{S P}$ denotes elastic shear buckling stress of the stiffened plate. It is important to emphasize that the curves depicted by Figure 2 are valid for an arbitrary plate thickness, i.e. $t / B$ ratio.

Generally, it can be noted that the transverse stiffening represents better approach for the $L / B<1$ cases, while longitudinal stiffening is preferable for the $L / B>1$ cases. Furthermore, it should be noted that inverse pairs of the $L / B$ values (e.g. $L / B=2$ and $L / B=0.5$ ) are characterized by the identical results. This is due to the inherent irrelevance of the acting edge shear load direction, which consequently induces overall symmetry of the obtained results with respect to the $L / B=1$ case, which is characterized by the lowest attainable relative critical shear stress increase. For this case identical results are obtained by longitudinal and transverse stiffening, since both of those approaches result in an identical stiffened plate layout. Hence, based on the above considerations, it can be generally concluded that the plate stiffening with stiffeners parallel to the longer side of the initial plate always represents a more gainful approach, irrespective whether $L / B<1$ or $L / B>1$.

\subsection{Existing formulations of the stiffened plate elastic shear buckling capacity}

The first formulation regarding the considered problem was proposed by Timoshenko, see Bleich \& Ramsey (1952), for a stiffened plate having one or

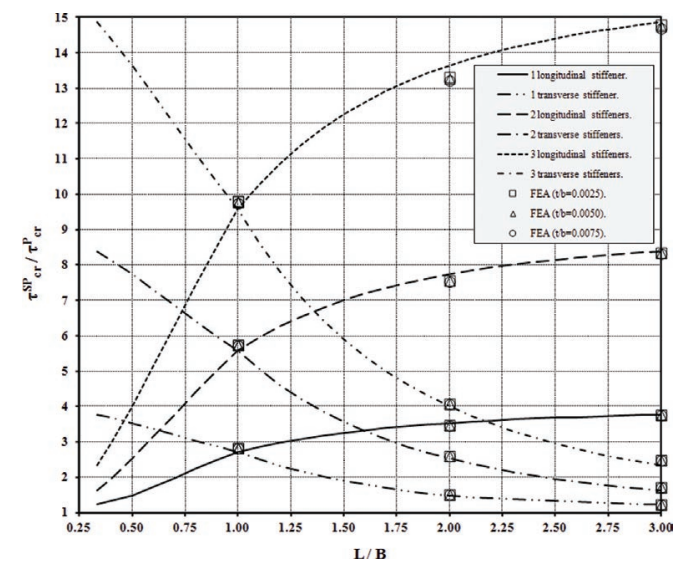

Figure 2. Maximum increase in the elastic shear buckling capacity attainable by the plate stiffening. two transverse stiffeners. This pioneering theoretical work based on the energy approach was extended subsequently by many authors and a more extensive overviews of the research work regarding this problem can be found in Bleich \& Ramsey (1952), Hughes (1988) and Alinia (2005). All of the existing formulations are based on the previously mentioned Timoshenko's paradigm and it is interesting to note that virtually all of them are derived considering stiffening parallel to the shorter side of the initial plate only, although the discussion given in Section 1.3 clearly suggests that an alternative stiffening approach is much more effective.

\section{MODELING OF THE CONSIDERED PROBLEM}

\subsection{Design of numerical experiments}

In order to validate the above given theoretical considerations, as well as to provide the basis for the general formulation proposed in Section 4, close to three hundred rationally designed numerical experiments are performed employing the Finite Element Method (FEM) simulations for generation of the results for various (un)stiffened plate configurations. All considered variants are characterized by the same (isotropic) material ( $E=206 \mathrm{GPa}$; $v=0.3$ ) and $B=3200 \mathrm{~mm}$. Furthermore, due to the previously mentioned symmetry of the considered problem, only variants characterized by $L / B \geq 1$ are considered. All other geometrical properties fall within the (relatively wide) range determined to cover the characteristic dimensions of the ship hull girder's flat single side plating between wing and bilge tanks of the existing and variously sized bulk carriers.

Unstiffened plates are analyzed in order to investigate the correspondence of the results obtained by utilization of (2) and by numerical simulations. For this purpose five different $L / B$ values $(1 ; 2 ; 3$; $6 ; 12)$ and five different $t / B$ values $(0.0025 ; 0.00375$; $0.005 ; 0.00625 ; 0.0075)$ are considered, resulting in a total of 25 numerical experiments.

Furthermore, in order to verify the theoretically obtained envelopes (see Figure 2) and their independence of $t$, analyses of the plates stiffened by one, two or three longitudinal or transverse stiffeners (of the infinite flexural rigidity) are performed for three different $L / B$ values $(1 ; 2 ; 3)$ and three different $t / B$ values $(0.0025 ; 0.005 ; 0.0075)$, resulting in a total of 54 numerical experiments.

Finally, in order to generate the experimental basis for the proposed formulation, analyses of the plates stiffened by longitudinal stiffeners (of the finite flexural rigidity) are performed. In 
this respect, previously mentioned Timoshenko's paradigm (which neglects torsional rigidity of stiffeners) enables arbitrary selection of the shape and scantlings of the employed stiffener profile, since only the stiffeners moment of inertia $I^{S}$ is relevant for its flexural rigidity. Among an infinite number of the possible stiffener profile variants characterized by the same particular $I^{S}$, a flangeless (flatbar) stiffener profile is selected for the further consideration. This choice is due to the irrelevance of the higher section modulus (offered by the flanged profiles) for the considered problem and since the flatbar stiffener offers the highest possible $I^{S}=t_{w} h_{w}{ }^{3} / 12$ for the particular stiffener cross sectional area, or in another words, provides attainment of the particular flexural rigidity with the least amount of the stiffener material. Furthermore, since the results $\left(\tau_{c r}^{S P} / \tau_{c r}{ }^{P}\right)$ are suggestively independent of $t$, the same value $(16 \mathrm{~mm})$ is used for the stiffener web thickness $t_{w}$ and $t$ of all variants considered within this experimental batch and various stiffener flexural rigidities considered are attained only by variation of the stiffener web height $h_{w}$. Thereby, 13 different stiffener flexural rigidities are considered for each of the three different $L / B$ values $(1 ; 2 ; 3)$ and three different $N_{S}$ values $(1,2,3)$, resulting in a total of 117 numerical experiments.

Throughout this paper, particular variant can be easily identified according to the assigned designation (e.g. AR1-T16-S1 L designates the plate with $L / B=1, t=t_{w}=16 \mathrm{~mm}$ and one longitudinal stiffener).

\subsection{Numerical simulations}

All executed numerical simulations are based on utilization of the FEM analysis of the discretized models of the considered (un)stiffened plate variants, whose bifurcation buckling load is determined by the eigenvalue analysis, as implemented within the employed FEMAP/NX Nastran (2010) computer program. Lanczos method is used for the eigenvalue extraction and the lowest (positive) eigenvalue is accepted as the quantitative representative of the relevant buckling mode.

\subsection{Discretized model}

All considered models are discretized by the twodimensional, quadrilateral, isoparametric finite elements with four nodes (CQUAD4) and six degrees of freedom (DoFs) at each node.

The imposed edge shear load is applied by means of the properly oriented nodal forces along the plate edges, as illustrated by Figure 1. Their magnitude is determined according to the expressions given in Figure 1, where $F_{n}{ }^{L}$ and $F_{n}{ }^{B}$ denote the absolute values of the nodal forces applied along the $L$ and $B$ edges, respectively. $F_{\text {corner }}^{L}$ and $F^{B}{ }_{\text {corner }}$ denote the absolute values of the components (parallel to the $L$ and $B$ edges, respectively) of the resultant nodal force applied at the plate corners (nodes coincident with the points $\mathrm{P}_{1}, \mathrm{P}_{2}$, $\mathrm{P}_{3}$ and $\mathrm{P}_{4}$ ), while $A_{L}$ and $A_{B}$ denote the cross sectional areas of the $L$ and $B$ edges, respectively. $F^{L}$ and $F^{B}$ denote the absolute values of the total forces applied along the $L$ and $B$ edges, respectively, while $n^{L}$ and $n^{B}$ denote the total number of nodes along the $L$ and $B$ edges, respectively. Indicated expressions for $F_{n}{ }^{L}, F_{n}{ }^{B}, F_{\text {corner }}^{L}$ and $F^{B}$ corner are derived in order to ensure the proper in-plane deformation of the corner finite elements, i.e. to ensure that straightness of the plate edges is fully retained in the deformed state. In this respect, it can be observed that $F_{n}{ }^{L} / F^{L}$ corner and/or $F_{n}{ }^{B} / F^{B}$ corner ratios should be equal to 4 .

In order to properly simulate deformation of the simply supported (un)stiffened plate models, nodal DoF constraints are applied as described by Table 1 , where 0 and 1 denote constrained and unconstrained nodal DoF, respectively. For the numerical simulations of the simply supported plates reinforced by the stiffeners of the infinite flexural rigidity, in addition to the constraints given by Table 1, vertical nodal displacements are constrained $\left(T_{z}=0\right)$ for all nodes along the plate to stiffener joint (line segment $\mathrm{P}_{6} \mathrm{P}_{7}$ in Figure 1).

In order to rationally determine an appropriate finite element mesh density for discretization of all considered FEM models, an extensive mesh convergence study is performed. For this purpose, two extreme values of $L / B(1 ; 12)$ and $t / B(0.0025$; $0.0075)$ are considered for both unstiffened and stiffened (by three stiffeners of the infinite flexural rigidity) plate variants, since the sensitivity of the results obtained for those cases should envelope the sensitivities characteristic for all other (un)stiffened plate variants considered by the above given plan of numerical experiments. For each of those eight (un)stiffened plate variants, ten different mesh density variants are considered, with 12,24 , 36, 48, 60, 72, 84, 96, 108 and 120 elements along the $B$ edges. Corresponding number of elements along the $L$ edges is unambiguously determined so as to keep the finite element aspect ratio equal to

Table 1. Applied constraints of nodal DoFs.

\begin{tabular}{lllllll}
\hline Node location & \multicolumn{7}{c}{ Nodal DoFs: } \\
(see Figure 1) & $T_{x}$ & $T_{y}$ & $T_{z}$ & $R_{x}$ & $R_{y}$ & $R_{z}$ \\
\hline $\mathrm{P}_{1} \mathrm{P}_{2} ; \mathrm{P}_{2} \mathrm{P}_{3} ; \mathrm{P}_{3} \mathrm{P}_{4} ; \mathrm{P}_{4} \mathrm{P}_{1} ;$ & 1 & 1 & 0 & 1 & 1 & 0 \\
$\mathrm{P}_{5}$ & 0 & 0 & 1 & 1 & 1 & 0 \\
All other plate nodes & 1 & 1 & 1 & 1 & 1 & 0 \\
\hline
\end{tabular}


1 for each mesh density variant. This results in a total of 80 additional numerical experiments considered within the framework of the mesh convergence study.

The results of the mesh convergence study are concisely given by Figure 3. It can be noted that the mesh density characterized by 96 elements along the $B$ edges gives a considerable relative reduction $(>35 \%)$ of the total number of DoFs $N_{D o F}$, while always providing a very small relative difference $(<0.15 \%)$ of the obtained results $\left(\tau_{c r}\right)$, all with respect to the finest mesh density. Consequently, this mesh density is used for all subsequently performed numerical simulations.
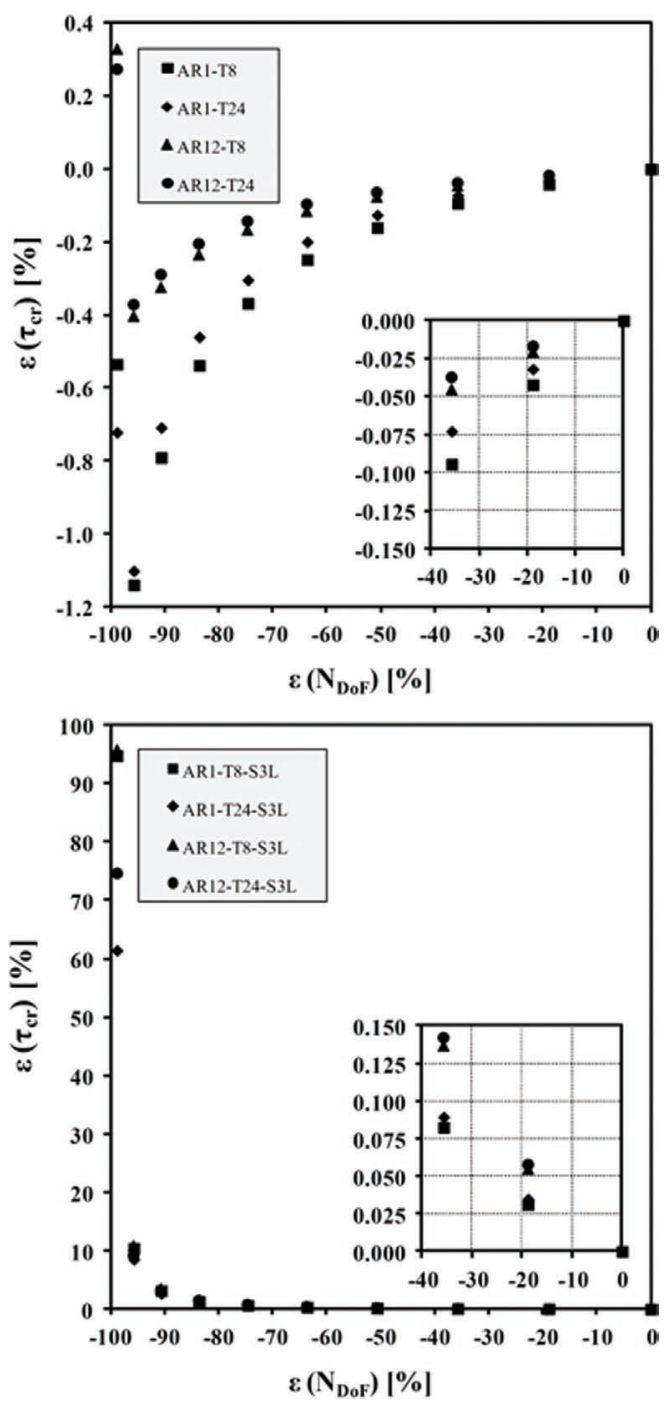

Figure 3. Results of the mesh convergence study.

\section{RESULTS}

Figure 4 illustrates the results obtained for the considered unstiffened plates. An excellent agreement of the results obtained by (2) and numerical simulations can be observed. Although an approximated relationship between those results is proposed (see Fig. 4), only a negligible error is introduced by its disregard $\left(\tau_{c r-F E A}^{P}=\tau_{c r-A N A L Y T I C A L}^{P}\right)$.

The results of the numerical simulations obtained for plates stiffened by the infinitely rigid stiffeners are given in Figure 2. It should be observed that the results obtained for various $t / B$ ratios are practically coincident. Furthermore, a very good agreement with the proposed theoretical envelopes can be observed.

Superimposed display of all results obtained by the numerical simulations performed for all considered plate variants reinforced by stiffeners with various (finite) flexural rigidity is given in $\tau_{c r}{ }^{S P} / \tau_{c r}^{P}-A^{S P} / A^{P}$ space, represented by Figure 5, where $A^{S P}$ denotes the area of the transverse cross section of the longitudinally stiffened plate, while $A^{P}$ denotes the area of the transverse cross section of the initial (unstiffened) plate. A more detailed display of the same results is given in $\tau_{c r}{ }^{P} / \tau_{c r}{ }^{P}-I^{S} / I^{b}$ space, represented by Figures 6-8, where $I^{b}=b t^{3} / 12$ represents the moment of inertia of the plating between stiffeners. Figure 6 additionally indicates the results obtained by three existing formulations of the considered problem, applicable only for $L / B=1$ cases among the considered ones. Figure 5 also displays the results obtained by the plate thickness increase approach in $\tau_{c r}^{P E} / \tau_{c r}^{P}-A^{P E} / A^{P}$ space, where $\tau_{c r}{ }^{P E}$ denotes the elastic shear buckling stress of the plate characterized by the (increased) equivalent thickness $t^{E}$ (see Fig. 5), while $A^{P E}$ denotes the area of its transverse cross section.

\section{ELASTIC SHEAR LOAD CAPACITY OF THE LONGITUDINALLY STIFFENED, SIMPLY SUPPORTED, FLAT PLATES MADE OF ISOTROPIC MATERIAL}

Results of the numerical simulations performed for nine considered plate variants reinforced by stiffeners of various (finite) flexural rigidity are used for formulation of the approximate description (surrogate model) of the considered problem. For this purpose a regression analysis based on utilization of the least squares method is performed, whereby coefficient of determination $\left(R^{2}\right)$ is used as a quantitative measure of the accomplished approximation quality.

The crucial choice regarding the appropriate form of the approximation function is based on the similar 'S-shaped' layout of the results obtained 

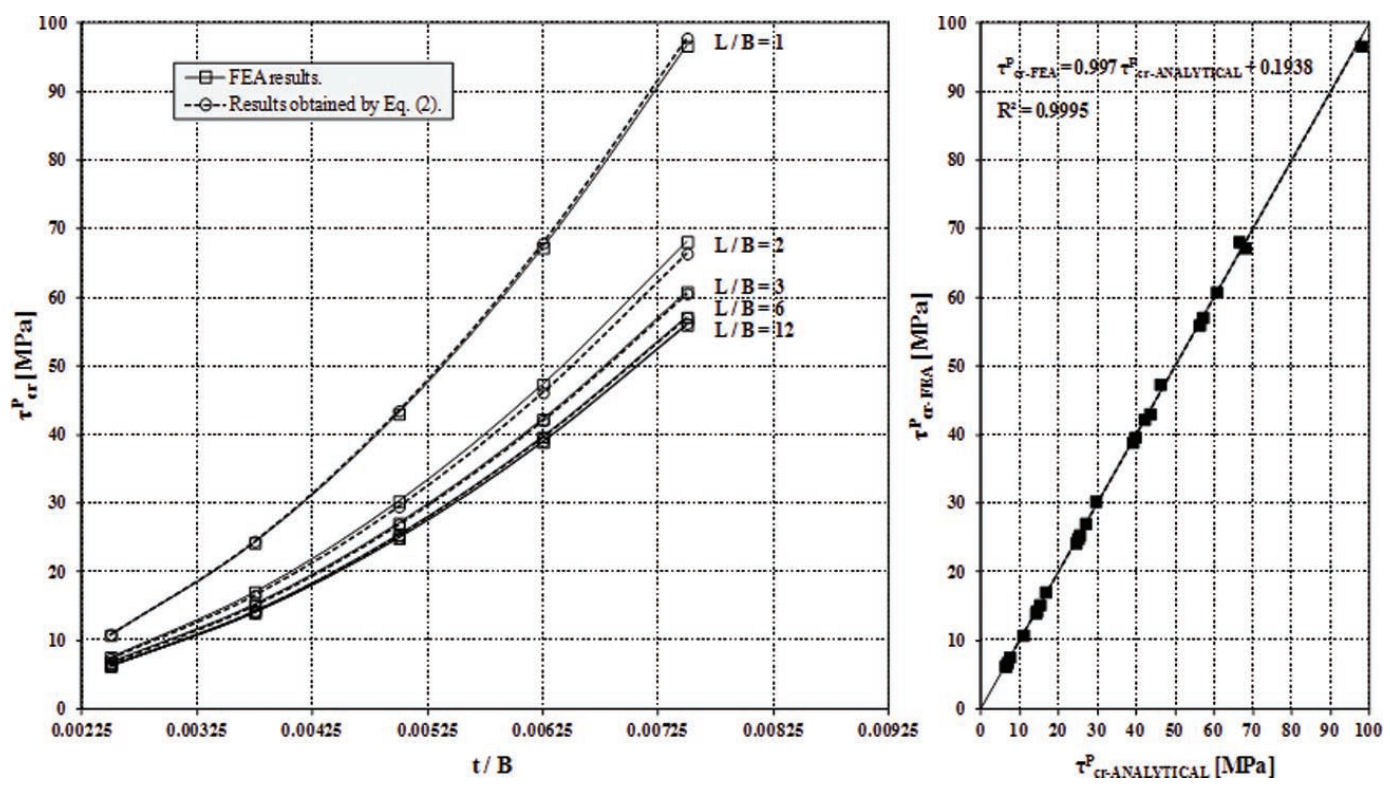

Figure 4. Unstiffened plate results, obtained by FEM analyses and by utilization of (2).

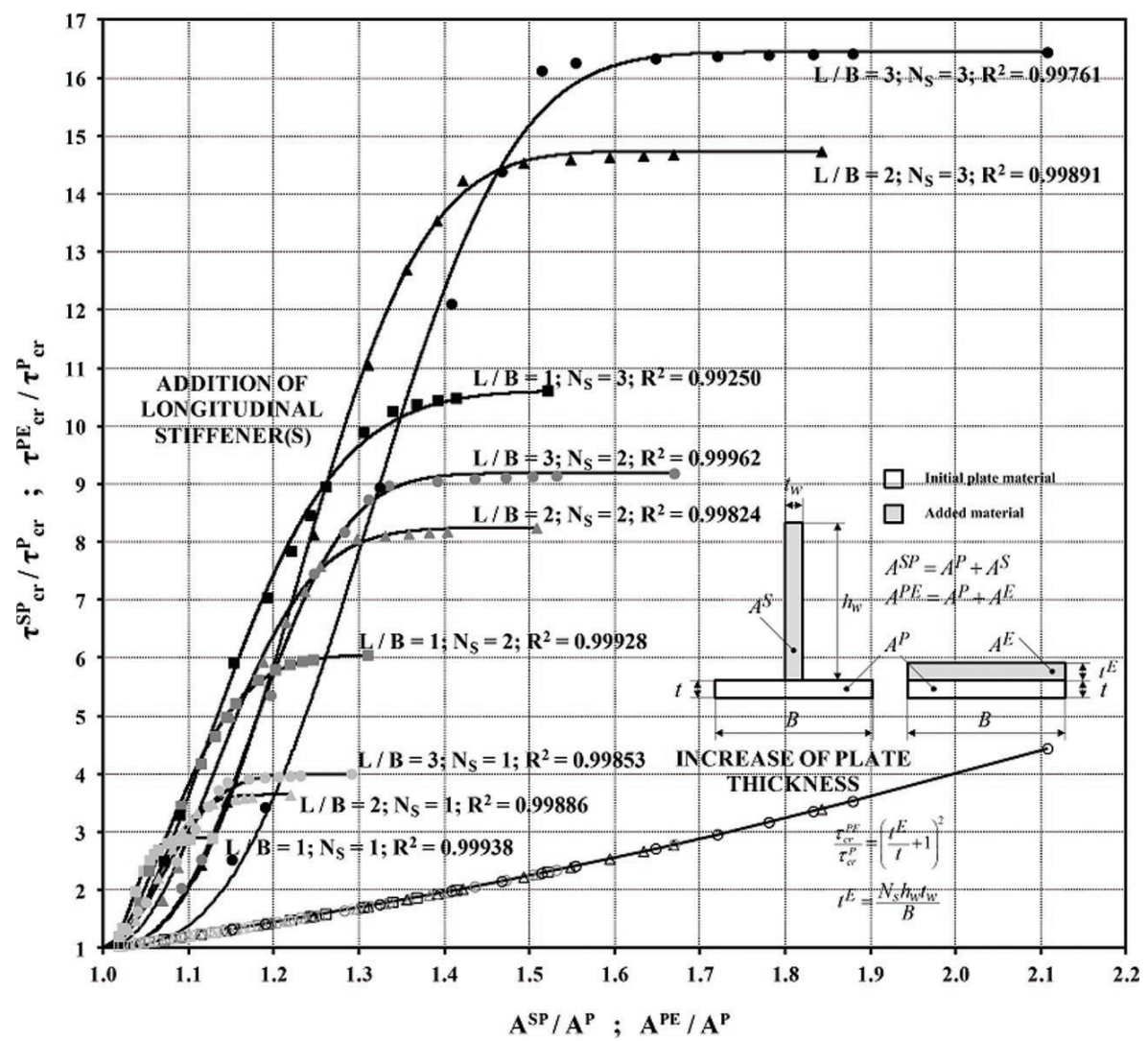

Figure 5. Results obtained for longitudinal stiffening and for increase of the plate thickness. 


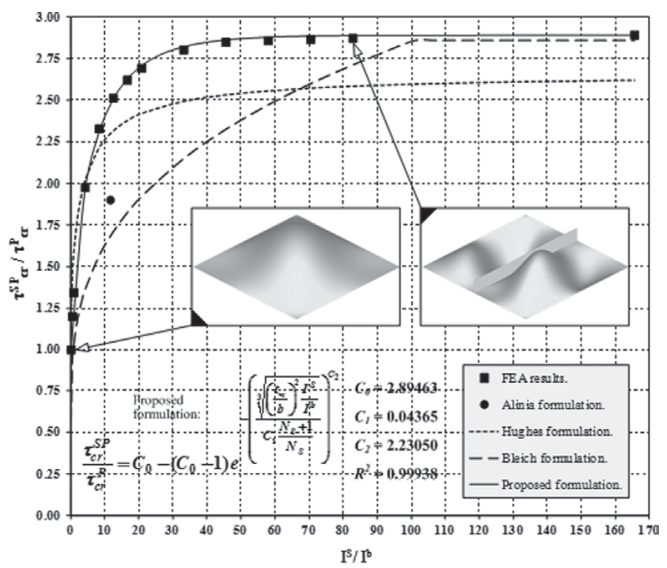

(a)

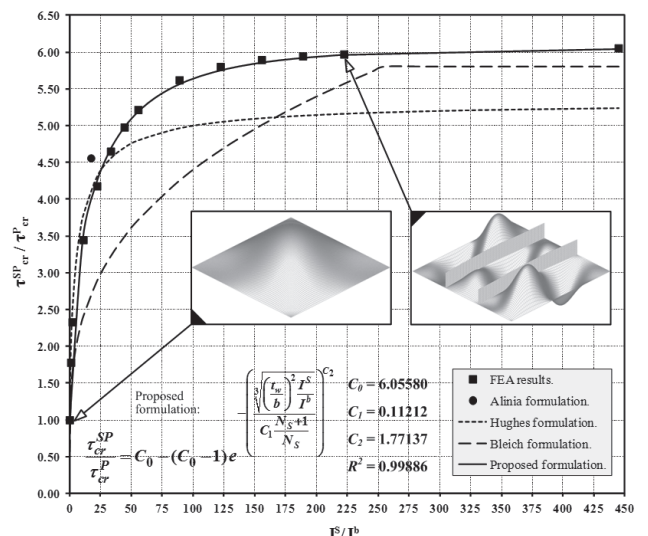

(b)

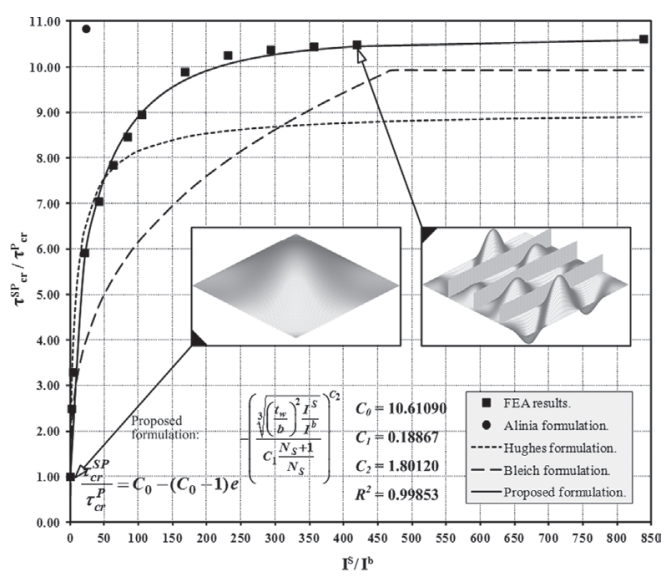

(c)

Figure 6. Results for AR1-T16: a) S1 L; b) S2 L; c) S3 L.

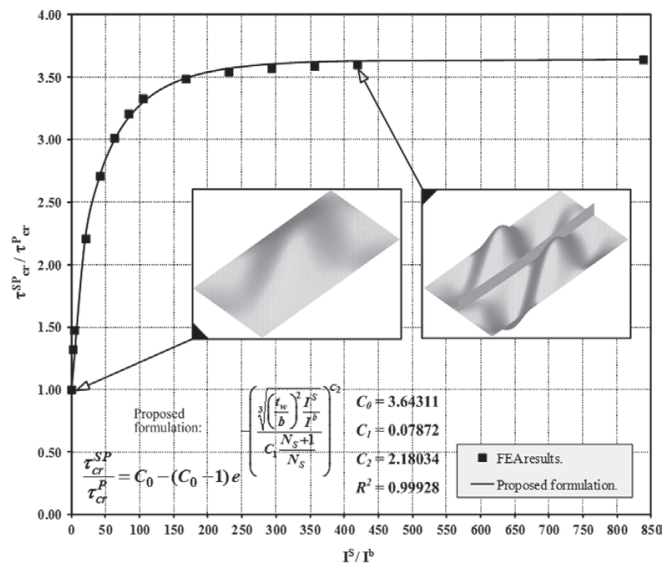

(a)

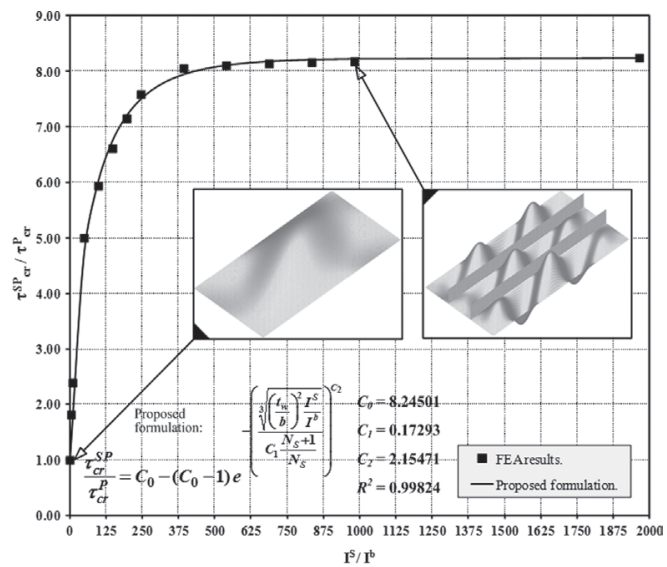

(b)

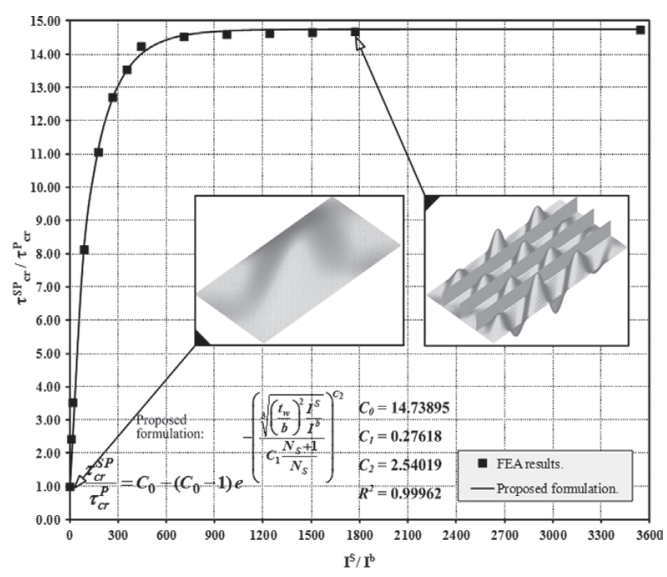

(c)

Figure 7. Results for AR2-T16: a) S1 L; b) S2 L; c) S3 L. 


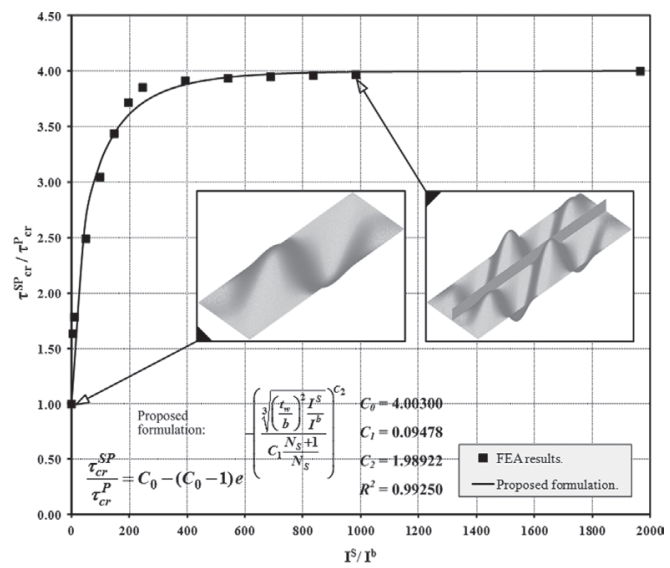

(a)

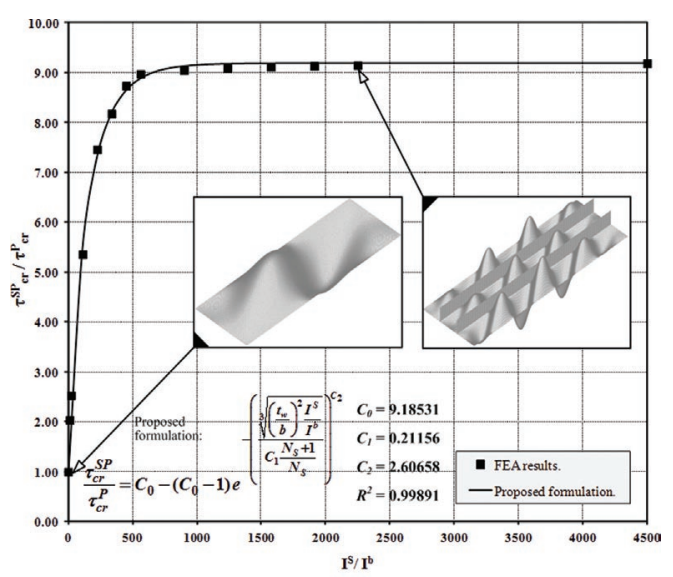

(b)

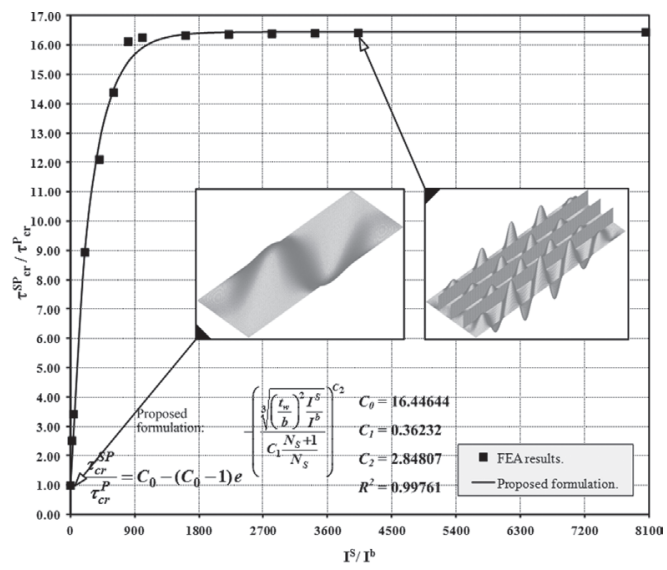

(c)

Figure 8. Results for AR3-T16: a) S1 L; b) S2 L; c) S3 L. for every considered stiffened plate variant, which can be observed in Figure 5. Although various sigmoid functions were considered for approximation of the results in $\tau_{c r}{ }^{S P} / \tau_{c r}{ }^{P}-A^{S P} / A^{P}$ space, the best approximate fit is accomplished by utilization of the adjusted cumulative distribution function of the Weibull distribution:

$$
\begin{aligned}
& \tau_{c r}^{S P} / \tau_{c r}^{P}=C_{0}-\frac{C_{0}-1}{\exp \left(\frac{A^{S P} / A^{P}-1}{C_{1}}\right)^{C_{2}}} \\
& \frac{A^{S P} / A^{P}-1}{C_{1}} \geq 0 ; C_{2}>0
\end{aligned}
$$

$C_{0}, C_{1}$ and $C_{2}$ represent the non-dimensional coefficients, whose specific values characterize every particular stiffened plate variant considered. $C_{0}$ actually represents the highest $\tau_{c r}{ }^{S P} / \tau_{c r}{ }^{P}$ value obtained (by numerical experiments) for each stiffened plate variant, while the corresponding values of $C_{1}$ and $C_{2}$ are determined numerically, using a Levenberg-Marquardt algorithm within the framework of the least squares method. Calculated values of the $C_{0}, C_{1}, C_{2}$ and $R^{2}$, along with the corresponding plots of (4) in $\tau_{c r}{ }^{S P} / \tau_{c r}{ }^{P}-I^{S} / I^{b}$ space, are given in Figures 6-8 for each of the nine considered stiffened plate variants, respectively.

In order to derive the general formulation valid for an arbitrary $L / B$ ratio within the considered range $(1 \leq L / B \leq 3)$, all values of the $C_{0}, C_{1}$ and $C_{2}$ determined for the nine considered stiffened plate variants are used. Considering the number of the available results and their scatter (see Fig. 9), the second degree polynomial is selected as an adequate function for determination of the exact fit:

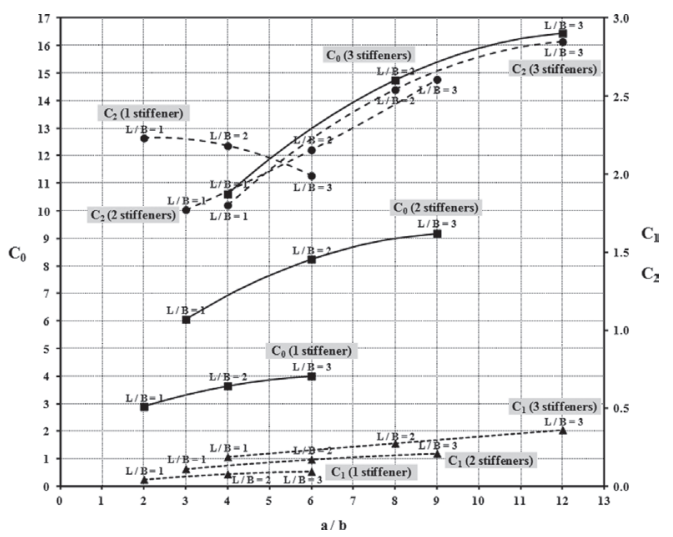

Figure 9. Graphical display of $C_{0}, C_{1}$ and $C_{2}$ coefficients. 
Table 2. Calculated values of $\left(\kappa_{i j}\right)_{0},\left(\kappa_{i j}\right)_{1}$ and $\left(\kappa_{i j}\right)_{2}$ coefficients.

\begin{tabular}{|c|c|c|c|c|c|c|c|c|c|}
\hline & \multicolumn{3}{|c|}{$N_{S}=1(j=1)$} & \multicolumn{3}{|c|}{$N_{S}=2(j=2)$} & \multicolumn{3}{|c|}{$N_{S}=3(j=3)$} \\
\hline & $\left(\kappa_{i j}\right)_{0}$ & $\left(\kappa_{i j}\right)_{1}$ & $\left(\kappa_{i j}\right)_{2}$ & $\left(\kappa_{i j}\right)_{0}$ & $\left(\kappa_{i j}\right)_{1}$ & $\left(\kappa_{i j}\right)_{2}$ & $\left(\kappa_{i j}\right)_{0}$ & $\left(\kappa_{i j}\right)_{1}$ & $\left(\kappa_{i j}\right)_{0}$ \\
\hline$C_{0}(i=0)$ & 1.75756 & 0.66568 & -0.04857 & 2.61768 & 1.35419 & -0.06938 & 4.06229 & 1.93972 & -0.07564 \\
\hline$C_{1}(i=1)$ & -0.01043 & 0.03179 & -0.00238 & 0.02913 & 0.03136 & -0.00123 & 0.09979 & 0.02239 & -0.00004 \\
\hline$C_{2}(i=2)$ & 2.13970 & 0.08064 & -0.01762 & 1.45656 & 0.09352 & 0.00381 & 0.63110 & 0.34641 & -0.01347 \\
\hline
\end{tabular}

$C_{i}=\left(\kappa_{i j}\right)_{0}+\left(\kappa_{i j}\right)_{1}\left(\frac{a}{b}\right)+\left(\kappa_{i j}\right)_{2}\left(\frac{a}{b}\right)^{2}$

$i=0, \ldots, 2 ; \quad j=1, \ldots, 3$

where $\left(\kappa_{i j}\right)_{0},\left(\kappa_{i j}\right)_{1}$ and $\left(\kappa_{i j}\right)_{2}$ represent the (nondimensional) polynomial coefficients valid for the respective stiffened plate variant. Calculated values which determine curves illustrated by Figure 9, are given in Table 2.

It should be noted that the validity of the proposed formulation is limited on stiffening parallel to the longer side of the plate, with $N_{S}=1,2,3$, while both $1 \leq L / B \leq 3$ and $0.333 \leq L / B \leq 1$ ranges are covered due to the previously mentioned symmetry of the considered problem (with respect to $L / B=1)$. However, within the $0.333 \leq L / B<1$ range, $L$ and $a$ should be interchanged with $B$ and $b$, respectively.

\section{CONCLUSIONS}

Accuracy of the commonly and widely accepted formulation of the elastic shear buckling capacity of the unstiffened flat plates, given by (2), is verified by the results obtained by numerical simulations (see Fig. 4). Furthermore, proposed theoretical envelopes and their independence of $t$, derived using Timoshenko's paradigm and (2), are also verified by the obtained results of the numerical simulations (see Fig. 2). This confirms the proposition that longitudinal stiffening for $L / B>1$ cases and transverse stiffening for $L / B<1$ cases, i.e. stiffening parallel to the longer side of the plate, always represents a more effective approach to the considered problem than the stiffening parallel to the shorter side of the plate (considered by all existing formulations). Moreover, comparison of the results obtained for all considered plates reinforced by stiffeners of various (finite) flexural rigidity (see Figs. 5-8), with respect to the results obtained for the plate thickness increase approach (see Fig. 5), suggests that the proposed stiffen- ing approach always represents a more effective approach to the considered problem. Hence, it can be unconditionally concluded that the proposed stiffening approach represents the most rational course for the elastic shear load capacity magnification of the simply supported plates. Furthermore, based on the results of the rationally designed and properly configured numerical simulations, an approximate formulation for this stiffening approach is proposed (valid for $0.333 \leq$ $L / B \leq 3$ and $\left.N_{S}=1,2,3\right)$. In this respect, a considerably high accuracy level of the proposed formulation, as well as its convenient mathematical nature, can contribute to its recognition as an useful additional tool in the process of analysis and/ or design of the ship structures.

\section{ACKNOWLEDGEMENTS}

Thanks are due to our student Antonio Mikulic for realization of the portion of the structural models and numerical simulations performed within the scope of work presented by this paper. This work has been supported in part by Croatian Science Foundation under the project 8658.

\section{REFERENCES}

Alinia, M.M. 2005. A study into optimization of stiffeners in plates subjected to shear loading. Thin-Walled Structures 43: 845-860.

Bleich, F. \& Ramsey, L.B. 1952. Buckling strength of metal structures. New York: McGraw-Hill.

FEMAP/NX Nastran. 2010. Software Documentation. Siemens Product Lifecycle Management Software.

Hughes, O.F. 1988. Ship structuraldesign: A rationally-based, computer-aided optimization approach. Jersey City: The Society of Naval Architects and Marine Engineers.

Timoshenko, S.P. \& Gere, J.M. 1985. Theory of elastic stability. New York: McGraw-Hill.

Zhang, S., Kumar, P. \& Rutherford, S.E. 2008. Ultimate shear strength of plates and stiffened panels. Ships and Offshore Structures 3(2): 105-112. 